\title{
In-hive patterns of temporal polyethism in strains of honey bees (Apis mellifera) with distinct genetic backgrounds
}

\author{
Adam J. Siegel • M. Kim Fondrk • Gro V. Amdam • \\ Robert E. Page Jr.
}

Received: 19 May 2013 /Revised: 13 June 2013 / Accepted: 13 June 2013 /Published online: 14 July 2013

(C) The Author(s) 2013. This article is published with open access at Springerlink.com

\begin{abstract}
Honey bee workers exhibit an age-based division of labor (temporal polyethism, DOL). Younger bees transition through sets of tasks within the nest; older bees forage outside. Components of temporal polyethism remain unrevealed. Here, we investigate the timing and pattern of preforaging behavior in distinct strains of bees to (1) determine if a general pattern of temporal DOL exists in honey bees, (2) to demonstrate a direct genetic impact on temporal pacing, and (3) to further elucidate the mechanisms controlling foraging initiation. Honey bees selected for differences in stored pollen demonstrate consistent differences in foraging initiation age. Those selected for increased pollen storage (high pollen hoarding strain, HSBs) initiate foraging earlier in life than those selected for decreased pollen storage (low pollen hoarding strain, LSBs). We found that HSBs both initiate and terminate individual pre-foraging tasks earlier than LSBs
\end{abstract}

Communicated by M. Beekman

A. J. Siegel $(\bowtie) \cdot$ M. K. Fondrk $\cdot$ G. V. Amdam $\cdot$ R. E. Page Jr. School of Life Sciences, Arizona State University,

Tempe, AZ, USA

e-mail: adam.siegel@mail.huji.ac.il

M. K. Fondrk

Department of Entomology, University of California Davis, Davis, CA, USA

G. V. Amdam

Department of Chemistry, Biotechnology and Food Science,

Norwegian University of Life Sciences, Aas, Norway

R. E. Page Jr.

College of Liberal Arts and Sciences, Arizona State University,

Tempe, AZ, USA

Present Address:

A. J. Siegel

Department of Ecology, Evolution and Behavior, Alexander

Silberman Institute of Life Sciences, The Hebrew University,

Jerusalem, Israel when housed in a common hive environment. Unselected commercial bees (wild type) generally demonstrated intermediate behavioral timing. There were few differences between genotypes for the proportion of pre-foraging effort dedicated to individual tasks, though total pre-foraging effort differences differed dramatically. This demonstrates that behavioral pacing can be accelerated or slowed, but the pattern of behavior is not fundamentally altered, suggesting a general pattern of temporal behavior in honey bees. This also demonstrates direct genetic control of temporal pacing. Finally, our results suggest that earlier HSB protein (pollen) consumption termination compared to LSBs may contribute to an earlier decline in hemolymph vitellogenin protein titers, which would explain their earlier onset of foraging.

Keywords Honey bee worker - Temporal polyethism . Division of labor $\cdot$ Pre-foraging behavior $\cdot$ Behavioral pacing $\cdot$ Behavioral pattern

\section{Introduction}

Eusocial insects demonstrate a division of labor (DOL) with behavioral task specialization, which is believed to be a prime enabler for this group's ecological success (Oster and Wilson 1978). Therefore, DOL in eusocial insects has been a central theme of evolutionary studies since Darwin (Darwin 1859). Because of their highly organized DOL and economic importance, honey bees are an often studied model social system (Winston 1987; Graham et al. 1992; Seeley 1995; Page et al. 2006). Reproductive DOL in honey bees is demonstrated by anatomically distinct reproductive queens and facultatively sterile female workers (Winston 1987). Among the workers, there is an age-correlated behavioral DOL, referred to as temporal polyethism. Young workers perform brood-nest associated tasks such as brood-cell cleaning and larval feeding. Middle-aged bees typically 
perform food processing, nest construction, and guarding. Finally, older bees progress to foraging outside the nest for food (Lindauer 1952; Seeley 1982; Winston 1987; Seeley and Kolmes 1991; Johnson 2003, 2008, 2010). The final shift from within-nest tasks to foraging is one of the most easily recognized and commonly studied transitions and is often used as a benchmark measure for the pacing of temporal polyethism. However, prior behavioral transitions are also essential to colony function and may have an impact on the transition to foraging (Seeley 1982; Calderone and Page 1991; Seeley and Kolmes 1991; Pankiw and Page 2001).

There is variation in the pacing of the hive to forager transition between individual bees, colonies, genotypes, and phenotypes (Calderone and Page 1988, 1991). At the group level, this may be limited to proportionally shortening or extending time spent performing within-nest tasks. Alternatively, some tasks could be skipped, disproportionately truncated or extended, or the order of task performance could be fundamentally different between genotypically distinct groups of honey bees. Johnson and Frost (2012) demonstrated that individual workers sometimes skip some tasks. Variation in pacing between distinct groups of bees could be due to direct genetic effects on the pacing of temporal polyethism. Pacing variation could also be due to indirect genetic effects caused by variation in nestmate genotypes within different colonies (Linksvayer and Wade 2005). These two possibilities are not mutually exclusive. For example, bee phenotypes such as individual dry mass and ovariole number are effected by interactions between direct and indirect genetic effects (Linksvayer et al. 2009).

The first aim of this study was to determine if there is a fundamental pattern to temporal polyethism across honey bee groups demonstrating different colony-level phenotypes. This information will lead to a better understanding of the constraints on temporal DOL. To address this aim, we investigated the order of, total time spent on, and proportion of time spent on within-nest tasks by genotypically and phenotypically distinct honey bee groups. Groups investigated were wild type (unselected commercial bees) and two strains of artificially selected honey bees that demonstrate predictable differences in foraging onset. If the foraging onset differences between these phenotypic groups result from proportional differences in pre-foraging task performance, this would suggest a general temporal behavioral program that can be accelerated or slowed down but not profoundly altered. Alternatively, disproportionate pre-foraging task performance extension, truncation, or task skipping between these groups would demonstrate that temporal polyethism is highly flexible and can be fundamentally different between isolated honey bee groups. This would suggest no general temporal pattern of behavior in honey bees.

The selected strains used to investigate the pre-foraging patterns of temporal behavior in honey bees were the high (HSBs) and low (LSBs) pollen hoarding strains of Page and
Fondrk (1995). Page and Fondrk selected for the amount of surplus pollen stored in combs by colonies creating the HSBs and LSBs. Selection was based on the methods developed by Hellmich et al. (1985). At the time of this study, HSBs and LSBs had undergone selection for 26 generations over 14years with out-crossing every third generation. Both selection programs, Hellmich et al. (1985) and Page and Fondrk (1995), resulted in behavioral syndromes related to foraging. HSBs from both selection programs collected and stored more pollen and foraged earlier in life than LSBs (Calderone and Page 1988; Pankiw and Page 2001). In addition, Page and Fondrk (1995) found that HSBs are more sensitive to sucrose than LSBs and are willing to accept nectar of a lower sugar concentration (Pankiw and Page 1999). It is important to note that in both selection programs, founding queens originated from commercially available stocks and that these observed behavioral relationships are present in non-selected, commercially available, wild-type bees.

HSBs from generation 7 of Hellmich et al. (1985) foraged approximately 1 day earlier than LSBs (Calderone and Page 1988). Calderone and Page (1991) conducted a study of preforaging behavior of bees from generation 8 of the Hellmich et al. (1985) strains and found few behavioral differences between them, consistent with the small difference in foraging onset. By generation 11, HSBs of Page and Fondrk (1995) were foraging as many as 12 days earlier in life than LSBs (Pankiw and Page 2001). Amdam et al. (2006, 2010) have demonstrated that differences in the foraging behavioral syndrome are controlled by developmental processes that begin prior to the onset of adult life.

The second aim of this study was to confirm a direct genetic effect on the pacing of temporal polyethism in honey bees. This aim was met by co-fostering bees of the three distinct genetic backgrounds in the same colonies. This procedure eliminated all potential environmental effects (e.g., brood presence, food storage, forager number; Huang and Robinson 1992, 1996; Johnson 2003, 2010) as well as indirect genetic effects (e.g., different levels of queen mandibular pheromone, which can repress foraging genes and delay foraging onset; Grozinger et al. 2003). Any observed differences between the distinct groups must therefore be due to genotypic differences between the HSBs, LSBs, and wild-type bees, and not environmental or indirect genetic effects.

The third aim of this study was to bridge the gap between development and foraging onset. We attempted to meet this aim by comparing the protein feeding dynamics between the HSBs and LSBs. Vitellogenin ( Vg), a behavioral affecter protein, interacts with juvenile hormone to play a regulatory role on foraging initiation (Amdam et al. 2003, 2007; Nelson et al. 2007). Significant differences in juvenile hormone levels between HSBs (early foraging onset) and LSBs (late foraging onset) bees begin during larval development (Amdam et al. 2010). Vg titers are high in young workers 
and decrease as they age (Rutz and Luscher 1974). Nelson et al. (2007) demonstrated that reduced Vg stimulates the onset of foraging. HSBs demonstrate both an earlier drop in $\mathrm{Vg}$ titers and a correspondingly earlier foraging onset compared to LSBs (Amdam et al. 2007). In young nurse bees, some of the protein (in the form of pollen) consumed (Crailsheim et al. 1992; Hrassnigg and Crailsheim 1998) is converted to $\mathrm{Vg}$ and incorporated into the brood food (proteinous glandular secretions produced by the hypopharyngeal glands). The brood food is then fed to developing larvae (Amdam et al. 2003). The earlier drop in Vg and initiation of foraging observed in HSBs could be facilitated by either increased brood feeding, thereby depleting circulating $\mathrm{Vg}$, or earlier termination of pollen consumption by HSBs compared to LSBs

Here, we describe the pre-foraging dynamics of the Page and Fondrk (1995) HSBs and LSBs. We conducted an observation hive study comparing the age of transition through a series of tasks between HSBs, LSBs, and wild-type bees. We then constructed temporal polyethism schedules of the HSBs, LSBs, and wild-type bees, with particular attention paid to the analysis of behavior that could impact Vg quantity, including pollen consumption and brood feeding. Comparing the differences in the appearance and duration of the pre-foraging behavior of these strains will shed light on the fundamental nature of behavioral pacing in honey bees. Doing so in a common hive environment will confirm a direct genetic control of behavioral pacing. Additionally, investigating the protein feeding dynamics of the HSBs and LSBs will help bridge the gap between development and foraging onset.

\section{Materials and methods}

This research was conducted in June and July of 2005 at the University of California at Davis Bee laboratory. The daily activities of individual HSBs, LSBs, and unselected bees were observed over a 29-day period in a common hive environment.

\section{Source of bees}

Focal bees were derived from the 26th generation of bees selected for area of pollen stored in the combs (Page and Fondrk 1995). Measurements of stored pollen revealed that LSB and HSB colonies stored $298 \mathrm{~cm}^{2}$ of pollen $(n=21)$ and $1,049.2 \mathrm{~cm}^{2}(n=14)$, respectively, in the same comb area (Student's $t$ test, $p<0.0005$ ). Commercial bees that were located near UC Davis were used for controls. Bees from three source colonies of each strain were used in this study. Two additional colonies of commercial origin served as the source of background bees in the experiment.

\section{Bee preparation}

Combs of mature pupae from the source colonies were placed in an incubator $\left(34{ }^{\circ} \mathrm{C}, 50 \%\right.$ relative humidity) overnight. Three hundred newly emerged workers of each selected strain and the commercial controls were uniquely marked with plastic numbered tags (Honig Müngersdorff) glued to the thorax. A paint mark (Testors Enamel) was placed on the abdomens to differentiate bees from two experimental replicates and facilitate identification of tagged bees when their thoraces were obscured in a comb cell (Seeley 1982; Seeley and Kolmes 1991). Tagged workers were introduced to a four-frame observation hive (hereafter referred to as hive 1 , see below) within $12 \mathrm{~h}$ of emergence. The marking procedure was repeated $24 \mathrm{~h}$ later using 900 additional unique tags and abdomen marks, and the second group was introduced to a second four-frame observation hive (hereafter referred to as hive 2).

\section{Observation-hive colony setup}

Two commercial colonies (not of the HSB or LSB strains) were transferred to two glass-walled four-frame observation hives and placed in a windowless observation-hive shelter 67 days before the introduction of the tagged experimental bees. As has been used in previous studies, the unselected observation-hive colonies each had a laying queen and had adult workers covering both sides of three combs (approximately 4,500-5,000 workers), approximately $2.25-2.50$ combs of brood in all stages of development, $0.50-0.75$ combs of pollen, and $1.0 \mathrm{comb}$ of honey (Calderone and Page 1988). A runway, with a glass top to allow for observations of exiting and returning bees, connected the observation hive with the outside of the observation structure. Petroleum jelly was applied on both ends on the inside of the glass bridge cover to minimize the number of bees walking upside down.

\section{Nest activity observations}

Daily visual observations of pre-foraging honey bee behavior were conducted for a random sample of bees from each of the three tagged groups. Observed honey bee tasks were identified by the observer and categorized according to a comprehensive behavioral catalog, derived from multiple sources (Seeley 1982, 1995; Winston and Punnett 1982; Kolmes 1985; Robinson 1987; Calderone and Page 1991, 1996; Seeley and Kolmes 1991) and author observations (Table 1). Observations were conducted on 26 days over a 29-day period beginning on the third day of adult life for the bees in hive 1 and the second day of adult life in hive 2. Data were not recorded on three non-consecutive days during the observation period. 
Table 1 Behavioral catalog including task codes and descriptions

\begin{tabular}{|c|c|c|}
\hline Task & Code & Description \\
\hline Cell cleaning & $\mathrm{CC}$ & $\begin{array}{l}\text { Removing debris from used brood cells (cocoons, larvae excretion), cleaning cell walls. } \\
\text { Takes place in a cell not currently being used }\end{array}$ \\
\hline General nest sanitation & NS & Removing debris from nest (moldy pollen, old cappings, dead brood, and dead adults) \\
\hline Brood care & $\mathrm{BC}$ & Feeding larvae (head in brood cell $>1.3 \mathrm{~min}$ ), attending queen \\
\hline Construction & $\mathrm{CT}$ & $\begin{array}{l}\text { Smoothing wooden hive parts with mandibles and manipulating wax and } \\
\text { propolis in cracks and corners of the hive }\end{array}$ \\
\hline Fanning wings & $\mathrm{FA} / \mathrm{FAe}$ & Flapping wings while standing in hive/at entrance \\
\hline Food care & FC & Insertion of head into a cell containing nectar, receiving nectar-on bridge \\
\hline Grooming a nestmate & GG & Running nestmate body parts through mandibles \\
\hline Grooming self & GS & Running own body parts through mandibles \\
\hline Inspecting a cell & IC & The momentary insertion of the anterior portion of the head into an empty cell \\
\hline Nest care & $\mathrm{NC}$ & Manipulating wax of cells (not cappings), building new empty cells \\
\hline Patrolling & PT & Walking around nest \\
\hline Standing and chaining & ST & Standing stationary or hanging on nestmates while stationary (lack of activity) \\
\hline Brood cap manipulation & TB & Trimming or smoothing wax cappings on brood cells and capping brood with wax \\
\hline Honey cap manipulation & TH & Trimming or smoothing wax cappings on cells of honey and capping honey with wax \\
\hline Trophallaxis & TR & $\begin{array}{l}\text { Nestmate exchange of food (not near entrance), receiver thrusts tongue at donators } \\
\text { mouthpart, donator opens mouthparts pushes tongue forward, } \\
\text { and regurgitates a drop which is lapped up }\end{array}$ \\
\hline Vibrating & VB & Fast rhythmic body vibrations (non-dance) \\
\hline Head in pollen & HP & Insertion of head into a cell containing pollen \\
\hline Inspecting brood & IB & Head in brood cell, $<1.3 \mathrm{~min}$ \\
\hline Dancing & $\mathrm{DA} / \mathrm{DA}+$ & Dancing without/with pollen \\
\hline Washboarding/plaining & WA & Standing and rocking back and forth with mouthparts open \\
\hline Attending dance & $\mathrm{AD} / \mathrm{AD}+$ & Dance attendance without/with pollen \\
\hline
\end{tabular}

Daily pre-foraging behavioral observations took up to $7 \mathrm{~h}$ and were conducted between 8:15 AM and 4:20 PM. This time window was chosen to limit observations to direct daylight hours. Observations took place under low-light conditions using low-wattage commercial incandescent light bulbs. A small flashlight was used when necessary to see inside of comb cells. During observations, the entire area of the glass walls of the observation-hive colonies was overlaid with transparent plexiglass grids of 128 squares that were each approximately $60 \times 60 \mathrm{~mm}$. Each runway was overlaid with an additional transparent plexiglass grid of 20 (count) $-60 \times 60 \mathrm{~mm}$ squares. Each square was assigned a unique number. Half of the squares on both sides of each hive and the bridge were observed visually each day (138 squares per hive per day). This was the maximum number of squares that could be accurately observed on two observation hives during daylight hours. Cameras were not used, as it was impossible to see inside of comb cells to determine their contents on video recordings. A randomized sequence generator (Haahr and Haahr 1998) was used to generate a random list of the squares to be observed each day. No square was observed twice on a given day. Observations were performed on one comb and one side at a time for convenience and to allow for more data to be collected in a given period of time. The daily order of hive, hive-side, and comb was determined by a coin toss. For each square observation, the tagged bee nearest to the center of the observed square was determined. Each square was observed for 3-5 min to determine the behavior displayed by the focal bee and to wait for the focal bee to exit a comb cell if necessary to complete the observation. For the focal bee, the bee identification code, behavior code from Table 1, and in-hive location were determined and recorded. To avoid bias, the unique tag identification codes assigned to each genetic strain were not revealed to the observer until after all data were collected. Some bees were recorded multiple times over the course of the experiment.

Previous studies of this kind have used either a large daily sample technique similar to the technique utilized here (Seeley 1982; Calderone and Page 1991). Other studies have followed a small number of individual focal bees over their lifetime (Lindauer 1952). We chose the prior, as we were 
interested in determining and comparing general group phenotypes, not individual animal phenotypes.

Brood care (BC) and inspecting brood (IB) were combined for statistical analysis, as were nest care (NC) and construction (CT). The initiation and termination age for each task category was compared for the two strains and commercial control bees using a log-rank test. The first and last time a bee was observed performing a specific task was its initiation and termination age for the given task. The proportion of total preforaging effort that was dedicated to each individual task by each strain was calculated using the following procedure: First, the total number of times each individual was observed performing an individual task was summed. This sum was then divided by the total number of times the individual bee was observed performing all tasks. A Kruskal-Wallis test was then used to compare the proportions of the individuals for each task across the three strains. Mann-Whitney $U$ tests were used to make comparisons between the two selected strains. Non-parametric analyses were performed because these data were not normally distributed.

\section{Foraging activity observations}

Foraging behavior of marked bees was observed to determine at what age bees of each strain initiated foraging. Observations took place at the glass-topped bridge that connected the hive to the outside of the observation shelter. Prior to bridge observations, the outside of the hive was observed daily for $5 \mathrm{~min}$ to determine if marked bees were leaving the hive vicinity, or performing pre-foraging orientation flights in front of the hive. Orientation flights are easily recognized as workers flying in the vicinity of the hive in expanding circles (Winston 1987; Graham et al. 1992, pp. 296-298). No data were collected during time windows when bees were observed performing orientation flights.

Foraging data collection began when tagged bees were observed leaving the immediate vicinity of the hive. Twentyminute observations of bees leaving and returning were conducted on each hive every second day beginning on the ninth day of adult life and continuing to the end of the experiment. Because individual bees forage at different times throughout the day and some foraging trips last longer than $20 \mathrm{~min}$, observations were conducted at different times each day between 8:00 AM and 5:00 PM. The following information was collected for each bee: the bee identification code, whether it was leaving or returning to the hive, whether it was returning with or without pollen. If a bee returned carrying a pollen load, it was classified as a pollen forager. If a bee returned without a pollen load, it was classified as a non-pollen incoming bee. It is not possible to differentiate between nectar, water, or empty returning foragers without using destructive sampling. Workers that left and returned within 5 min were excluded from the forager category as a round trip of less than $5 \mathrm{~min}$ suggests an orientation flight (Sekiguchi and Sakagami 1966; Winston and Katz 1982; Robinson 1985). A contingency table $G$ test was used to compare strains for the proportion of bees returning with pollen. A Student's $t$ test was used to compare strains for the mean foraging initiation age. These data did meet the requirements for parametric analyses.

\section{Results}

As has been previously demonstrated, HSBs were more likely to return to the hive with pollen loads than were LSBs (Fig. 1; Page et al. 1998, 2006; Amdam et al. 2004, 2006). HSBs also initiated foraging at a significantly younger age (5.3-5.5 days younger depending on replicate) than LSBs (Fig. 2; Page et al. 1998, 2006). In contrast to findings by Calderone and Page (1991), we found several significant strain differences in preforaging behavior in addition to the expected differences in pollen collection and foraging initiation age. Self grooming, nest care, food care, manipulating brood comb, manipulating honey comb, brood care tasks, head insertion into pollen cells, and standing in the nest were frequently observed (refer to Table 1 for task descriptions). HSBs initiated and terminated several of these tasks earlier than LSBs, and wild-type bees generally demonstrated intermediate initiation and termination ages (log-rank test, $N_{\min }=11, N_{\max }=113, N_{\text {median }}=56.5$, Fig. 3). In both hive replicates, there were significant differences among the three groups tested for median initiation age for self grooming, patrolling, food care tasks, manipulating brood comb, and brood care tasks. In a single replicate, there were significant differences in initiation age for nest care tasks, manipulating honey comb, and standing (log-rank test, Fig. 3). In both replicates, there were significant differences among the three groups tested for median termination age for self grooming, nest care, patrolling, food care, and brood tasks. In a single replicate, there were significant differences in termination age for manipulating brood comb and manipulating honey comb. A two-way comparison of task groups between the HSBs and LSBs revealed additional differences in nest care initiation age (replicate 2: Mann-Whitney $U$ test, $Z=-2.19, P<0.05$ ) and manipulation of brood comb termination age (replicate 1 : Mann-Whitney $U$ test, $Z=-2.19, p<0.05$ ). These trends demonstrate a faster rate of transition between tasks in the HSBs and are consistent with their earlier foraging age.

Of particular note, when a comparison was performed between only HSBs and LSBs, the HSBs were shown to terminate the behavioral category "observed head in pollen (HP) cell" significantly earlier than the LSBs in one of the replicates, as would be predicted if the earlier drop in $\mathrm{Vg}$ observed in HSBs was a direct result of earlier termination of protein consumption (replicate 1: one-tailed Mann-Whitney $U$ test, $Z=-1.73, p<0.05$; replicate 2 : one-tailed MannWhitney $U$ test, $Z=-0.078, p>0.05$ ). 
Fig. 1 Number of foragers/ incoming bees of each strain returning with and without pollen. Replicate 1 (top); replicate 2 (bottom). HSBs are more likely to collect pollen than LSBs (contingency table $G$ test, ${ }^{*} p<0.05,{ }^{*} p<0.01$, $* * * p<0.001, N S$ not significant)
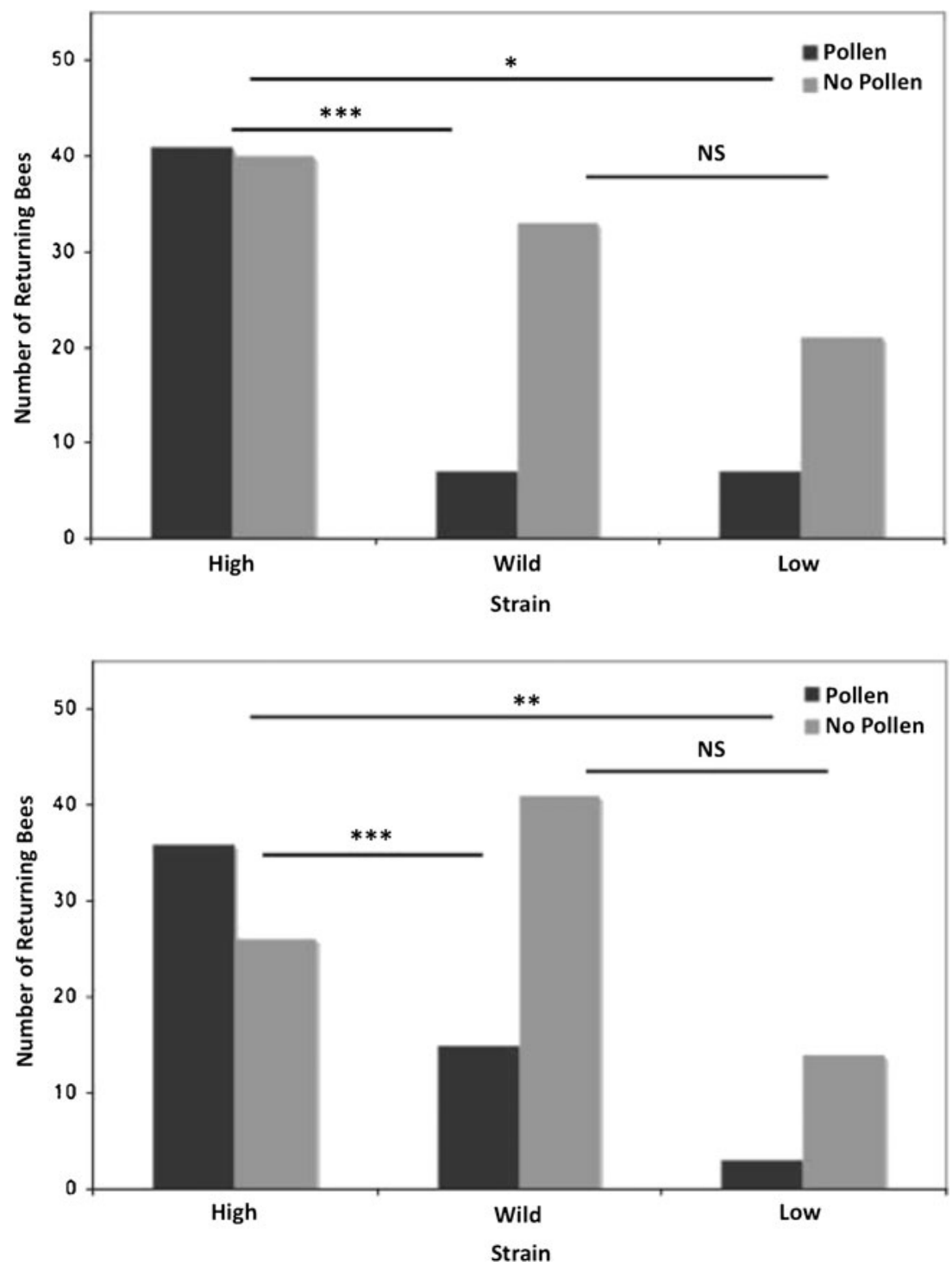

While there were differences between the strains in initiation and termination age, there were few significant differences for the proportion of pre-foraging effort dedicated to task groups between the strains (Kruskal-Wallis test, Fig. 4, refer to methods for full calculation procedure). Only one rarely observed task category, "manipulating honey comb" (TH) demonstrated a consistent difference in proportion across replicates. Of particular note is the lack of interstrain differences for BC (Fig. 4).

\section{Discussion}

HSBs initiated and terminated a majority of tasks earlier than LSBs (Fig. 3). This suggests a constant, faster rate of transition between tasks in the HSBs. This is consistent with the previous studies of behavioral transition rates of bees independently selected for pollen storage (Calderone and Page 1988, 1991), as well as the earlier foraging age demonstrated by HSBs compared to LSBs (Page et al. 1998, 2006; Amdam et al. 2007). Wild-type bees tended to demonstrate a temporal phenotype that was intermediate to the HSBs and LSBs. In addition, there was frequently higher variability in the task initiation and termination ages in wild-type bees (Fig. 3). This was not a surprising result, as wild-type bees would be expected to have higher genetic diversity among nest mates than individuals in the selectively bred strains. This variation could be beneficial in under variable environmental conditions. Further, in all groups, brood tasks $(\mathrm{BC}+\mathrm{IB})$ were conducted earlier than middle age tasks (e.g., $\mathrm{FC}$ - food processing and $\mathrm{NC}+\mathrm{CT}$-nest maintenance), as is predicted by the "middle-age worker" model (Seeley 1982; Johnson 2003, 2008, 2010). 
Fig. 2 Mean (+SE) foraging initiation age for bees of each strain. Replicate 1 (top); replicate 2 (bottom) 2. HSBs forage earlier than LSBs in both replicates (Student's $t$ test, letters represent significant difference $p<0.0001$ )
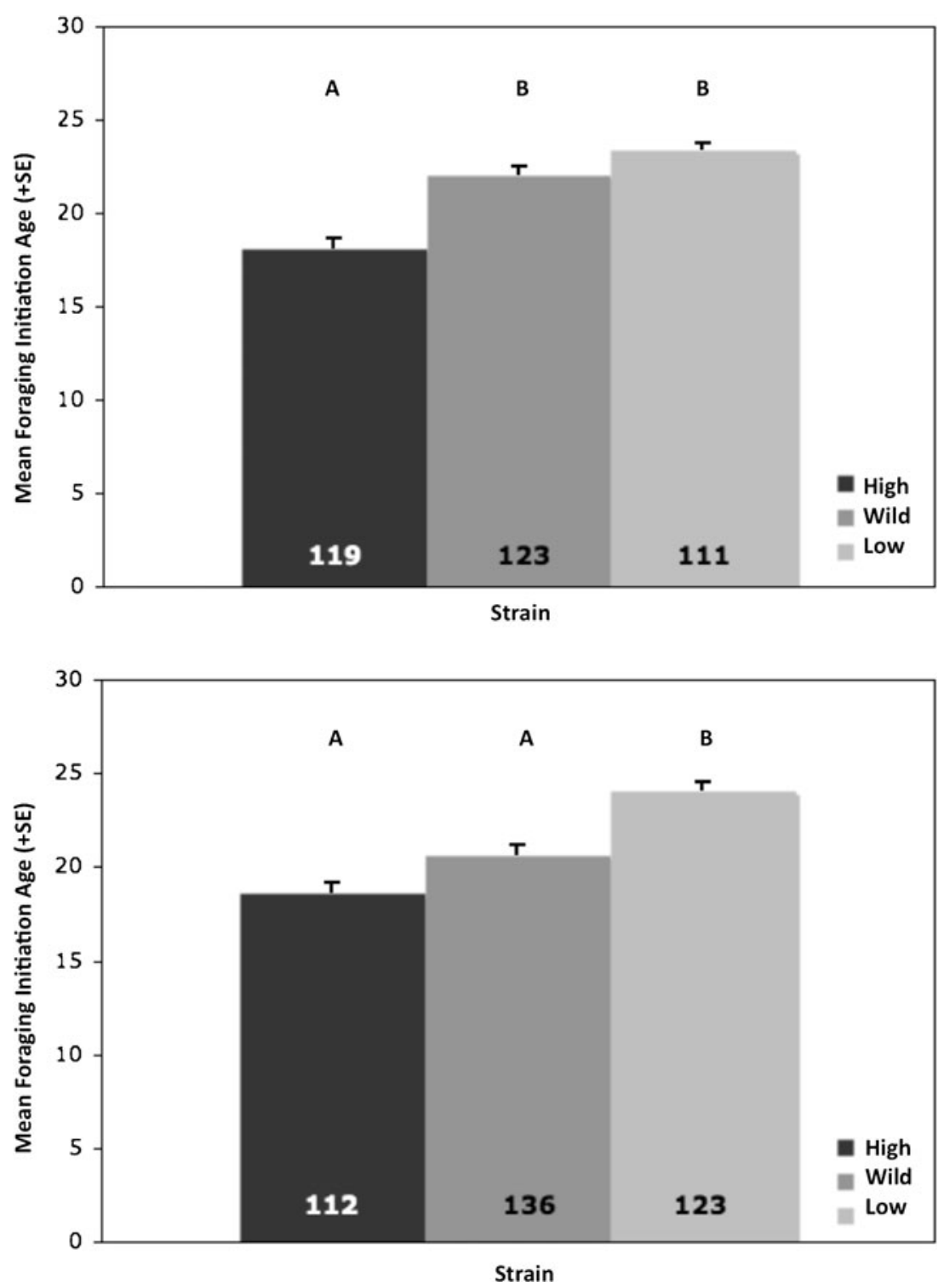

In contrast to the temporal polyethism findings, there were few significant differences for observed behavioral performance as a proportion of total within-nest activity. Additionally, though it has been previously demonstrated that some individual bees skip some tasks in the temporal polyethism (Johnson and Frost 2012), our results demonstrate that at the group level, all observed tasks were performed by all groups tested (both selected strains and the wild-type controls). This suggests that the task performance distribution requirements are similar across the strains and that observed differences are due to variation in the rate of task transition, rather than to changes in the order or proportion of effort associated with each task.

The results presented here support temporal polyethism as an organizational principal for task performance (see trend in Fig. 3). Genetically differentiated groups made transitions at different times even though they were the same age and shared a common hive environment (Calderone and Page 1988, 1991; Pankiw and Page 2001). This view was challenged by the "foraging for work" hypothesis of Tofts and Franks (1992). Tofts and Franks proposed that the apparent pattern of temporal polyethism was an artifact of young bees moving out of the central brood nest toward the periphery of the hive in search of tasks to perform, rather than the consequence of an innate behavioral pacer as is demonstrated by the data presented here.

Variable behavioral results between bee groups with distinct genetic backgrounds in the same hive environments demonstrate a direct genetic effect on behavioral pacing in honey bees. However, because the replicates were kept under the same conditions and were run simultaneously, we were not able to test for phenotypic plasticity in the focal groups. The direct genetic effect compliments, and likely interacts with, environmental (Huang and Robinson 1992, 1996; Johnson 2003, 
Fig. 3 Median common task initiation and termination age for bees from each strain. Replicate 1 (top); replicate 2 (bottom). Lefthand stars represent significant difference in initiation age. Right-hand stars represent significant difference in termination age. Refer to Table 1 for task codes. Note that the HSBs typically initiate and terminate tasks earlier than the LSBs with the wild-type bees falling intermediate. For some tasks with very short performance duration, a large number of individual bees were observed performing the task only one time each (e.g., HSBs$\mathrm{BC}$ in replicate 2: $N=82$ ). Note also the temporal trend between tasks represented by the dashed trend lines (log-rank test;

$N_{\text {min }}=11, N_{\text {max }}=113$,

$N_{\text {median }}=56.5 ; * p<0.05$,

$* * p<0.01, * * * p<0.005$ $* * * * p<0.001, * * * * * p<0.0001)$
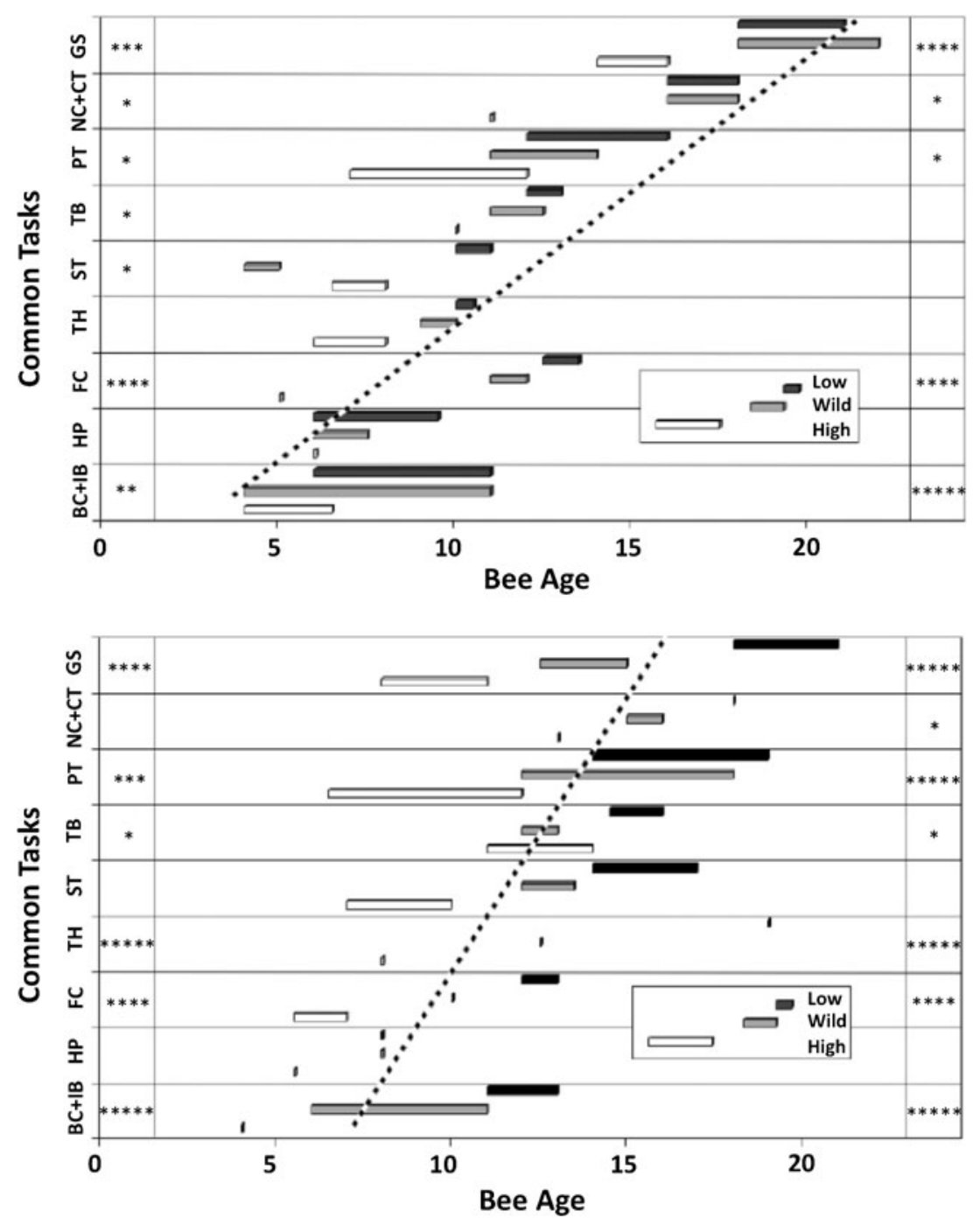

2010) and indirect genetic effects (Grozinger et al. 2003) on temporal pacing.

Finally, our results regarding protein consumption dynamics were inconclusive. However, they do suggest a mechanism through which protein consumption dynamics may have a regulatory affect on foraging initiation age which warrants further testing. An earlier decrease in Vg in HSBs compared to LSBs has been demonstrated and shown to be associated with earlier foraging initiation (Amdam et al. 2007; Nelson et al. 2007). Two non-mutually exclusive hypotheses can explain the faster rate of $\mathrm{Vg}$ decline in HSBs. First, the faster decrease in $\mathrm{Vg}$ titers observed in HSBs could be due to a higher proportion of their pre-foraging time spent feeding brood food to larvae compared to the LSBs. Second, the faster decrease in Vg titers might be a result of earlier termination of pollen consumption in HSBs compared to the LSBs. Pollen consumption is a primary source for protein in young workers (Crailsheim et al. 1992; Hrassnigg and Crailsheim 1998). Much of the consumed protein is converted to $\mathrm{Vg}$, which is a major component of the protein rich jelly used to feed larvae (Amdam et al. 2003); therefore, feeding larvae depletes circulating $\mathrm{Vg}$.

There was no difference in the proportion of time spent on brood care tasks between the HSBs and LSBS, showing that the earlier decrease in $\mathrm{Vg}$ in the HSBs is unlikely to be due to increased larval feeding. A two-way comparison between the HSBs and LSBs for the behavioral category "observed HP cell" suggests that the HSBs terminated pollen consumption earlier in both of the replicates, though statistically significant in only one replicate. There were few observations of "head in pollen cells," decreasing the chance of seeing a significant response. However, it is clear that the HSBs transitioned out of the brood nest and away from stored pollen much earlier than LSBs. This is shown by the temporal transition from feeding and inspecting brood to food care tasks. These are tasks performed away from the brood and stored pollen. This result suggests that earlier termination of pollen consumption is a possible contributing factor to the earlier decrease in Vg titers observed in HSBs and their subsequent earlier foraging. 
Fig. 4 Proportion of the total observations, bees of each strain were recorded performing common tasks. Each shade of gray represents a different task. "Other" category represents pooled observations of infrequently performed tasks (tasks listed in Table 1 that are not individually represented here). Replicate 1 (top); replicate 2 (bottom). Most tasks show that no difference in the proportion of total records individuals was observed performing each task. Refer to Table 1 for task codes (Kruskal-Wallis test; $N_{\min }=11$, $N_{\text {max }}=113, N_{\text {median }}=56.5$; ${ }^{*} p<0.05,{ }^{*} p<0.01$, $* * * p<0.001$ )

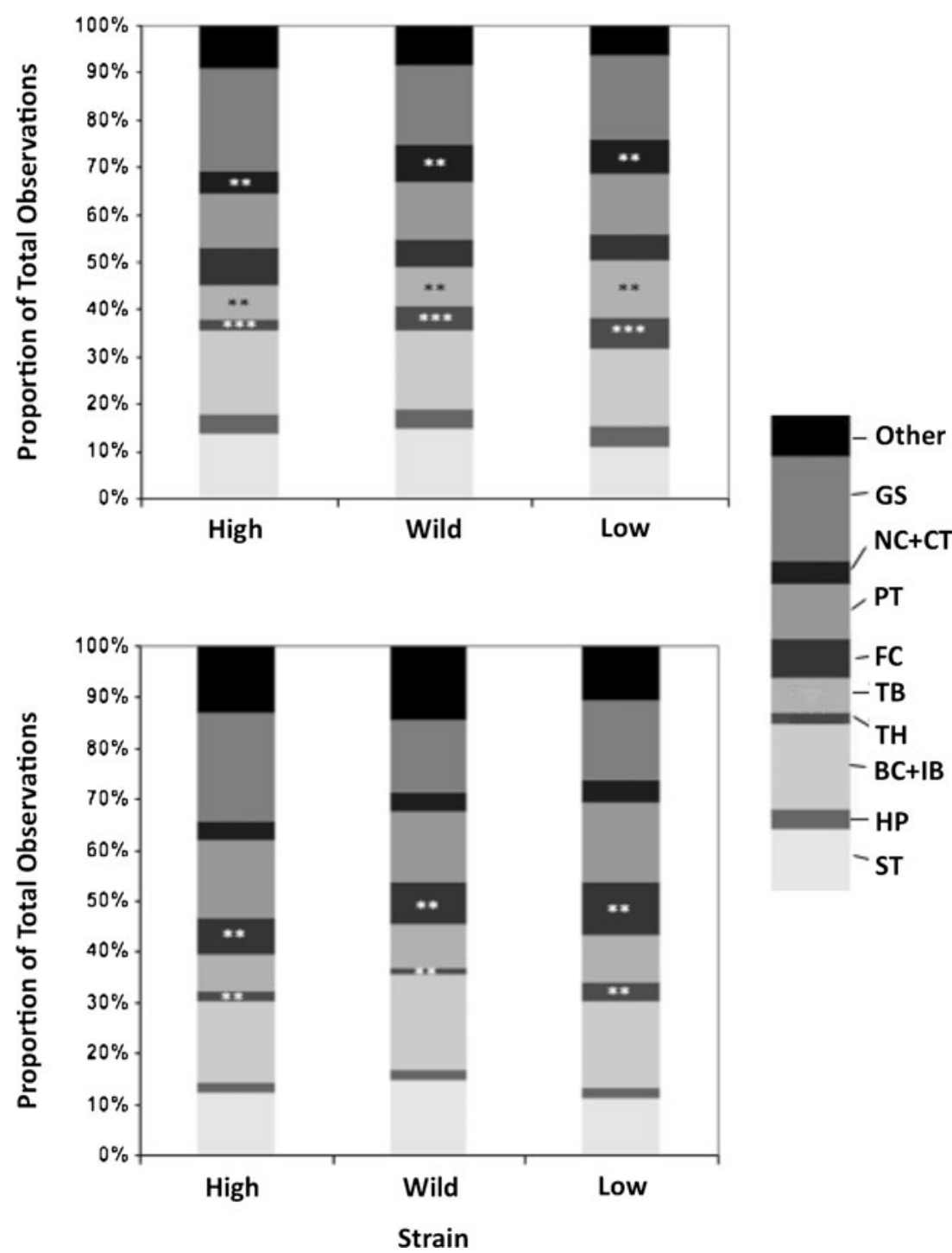

impacting temporal pacing. Finally, the data hints at a possible mechanism for the observed differences in timing of the onset of foraging involve the timing of cessation of pollen consumption, thereby reducing circulating titers of vitellogenin.

Acknowledgments The authors thank Andrew Hamilton, Colin Brent, Kevin McGraw, and Kate Ihle and five anonymous reviewers for comments and suggestions; Kate Ihle and Jennifer Tsuruda for help tagging bees; and Greyson Howard for hosting AJS in Davis, California during data collection. This project was funded by a grant from the National Institute of Health to REP. This research complies with all current laws of the United States of America and meets the ethical standards of Arizona State University.

Conflict of interest The authors declare that they have no conflict of interest.

Open Access This article is distributed under the terms of the Creative Commons Attribution License which permits any use, distribution, and reproduction in any medium, provided the original author(s) and the source are credited. 


\section{References}

Amdam GV, Norberg K, Hagen A, Omholt SW (2003) Social exploitation of vitellogenin. P Natl Acad Sci USA 100(4):1799-1802. doi:10.1073/Pnas.0333979100

Amdam GV, Norberg K, Fondrk MK, Page RE (2004) Reproductive ground plan may mediate colony-level selection effects on individual foraging behavior in honey bees. P Natl Acad Sci USA 101(31):11350-11355. doi:10.1073/Pnas.0403073101

Amdam GV, Csondes A, Fondrk MK, Page RE (2006) Complex social behaviour derived from maternal reproductive traits. Nature 439(7072):76-78. doi:10.1038/Nature04340

Amdam GV, Nilsen KA, Norberg K, Fondrk MK, Hartfelder K (2007) Variation in endocrine signaling underlies variation in social life history. Am Nat 170(1):37-46

Amdam GV, Page RE, Fondrk MK, Brent CS (2010) Hormone response to bidirectional selection on social behavior. Evol Dev 12(5):428-436. doi:10.1111/J.1525-142x.2010.00429.X

Calderone NW, Page RE (1988) Genotypic variability in age polyethism and task specialization in the honey bee, Apis mellifera (Hymenoptera: Apidae). Behav Ecol Sociobiol 22:17-25

Calderone NW, Page RE (1991) Evolutionary genetics of division-of-labor in colonies of the honey-bee (Apis mellifera). Am Nat 138(1):69-92

Calderone NW, Page RE (1996) Temporal polyethism and behavioural canalization in the honey bee, Apis mellifera. Anim Behav 51:631643

Crailsheim K, Schneider LHW, Hrassnigg N, Buhlmann G, Brosch U, Gmeinbauer R, Schoffmann B (1992) Pollen consumption and utilization in worker honeybees (Apis-mellifera-carnica) - dependence on individual age and function. J Insect Physiol 38(6):409-419

Darwin C (1859) On the origin of species by means of natural selection. Murray, London

Graham JM, Ambrose JT, Atkins EL, Avitabile A, Ayers GS, Blum MS et al (1992) The hive and the honey bee: a new book on beekeeping which continues the tradition of "Langstroth on the Hive and the Honeybee". Dadant \& Sons, Hamilton

Grozinger CM, Sharabash NM, Whitfield CW, Robinson GE (2003) Pheromone-mediated gene expression in the honey bee brain. P Natl Acad Sci USA 100:14519-14525. doi:10.1073/Pnas.2335884100

Haahr M, Haahr S (1998) Random.org. http://www.random.org. 2005

Hellmich RL, Kulincevic JM, Rothenbuhler WC (1985) Selection for high and low pollen-hoarding honeybees. J Hered 76:155-158

Hrassnigg N, Crailsheim K (1998) Adaptation of hypopharyngeal gland development to the brood status of honeybee (Apis mellifera L.) colonies. J Insect Physiol 44(10):929-939

Huang ZY, Robinson GE (1992) Honeybee colony integrationworker-worker interactions mediate hormonally regulated plasticity in division-of-labor. P Natl Acad Sci USA 89(24):1172611729. doi:10.1073/Pnas.89.24.11726

Huang ZY, Robinson GE (1996) Regulation of honey bee division of labor by colony age demography. Behav Ecol Sociobiol 39(3):147-158. doi:10.1007/S002650050276

Johnson BR (2003) Organization of work in the honeybee: a compromise between division of labour and behavioural flexibility. P R Soc B 270(1511):147-152. doi:10.1098/Rspb.2002.2207

Johnson BR (2008) Within-nest temporal polyethism in the honey bee. Behav Ecol Sociobiol 62(5):777-784. doi:10.1007/S00265-007-0503-2

Johnson BR (2010) Division of labor in honeybees: form, function, and proximate mechanisms. Behav Ecol Sociobiol 64(3):305-316. doi:10.1007/s00265-009-0874-7

Johnson BR, Frost E (2012) Individual-level patterns of division of labor in honeybees highlight flexibility in colony-level developmental mechanisms. Behav Ecol Sociobiol 66(6):923-930. doi:10.1007/S00265-012$1341-4$
Kolmes SA (1985) A quantitative study of the division of labor among worker honey bees. Z Tierpsychol 68(4):287-302

Lindauer M (1952) Ein Beitrag Zur Frage Der Arbeitsteilung Im Bienenstaat. Z Vergl Physiol 34(4):299-345

Linksvayer TA, Wade MJ (2005) The evolutionary origin and elaboration of sociality in the aculeate Hymenoptera: maternal effects, sibsocial effects, and heterochrony. Q Rev Biol 80(3):317-336

Linksvayer TA, Fondrk MK, Page RE (2009) Honeybee social regulatory networks are shaped by colony-level selection. Am Nat 173(3):E99-E107. doi:10.1086/596527

Nelson CM, Ihle KE, Fondrk MK, Page RE, Amdam GV (2007) The gene vitellogenin has multiple coordinating effects on social organization. Plos Biol 5(3):673-677. doi:10.1371/Journal.Pbio.0050062

Oster GF, Wilson EO (1978) Caste and ecology in the social insects. Monographs in population biology, vol 12. Princeton University Press, Princeton

Page RE, Fondrk MK (1995) The effects of colony level selection on the social-organization of honey-bee (Apis-mellifera L) colonies - colony level components of pollen hoarding. Behav Ecol Sociobiol 36(2):135144

Page RE, Erber J, Fondrk MK (1998) The effect of genotype on response thresholds to sucrose and foraging behavior of honey bees (Apis mellifera L.). J Comp Physiol A 182(4):489-500

Page RE, Fondrk MK, Hunt GJ, Guzman-Novoa E, Humphries MA, Nguyen K, Greene AS (2000) Genetic dissection of honeybee (Apis mellifera L.) foraging behavior. J Hered 91(6):474-479

Page RE, Scheiner R, Erber J, Amdam GV (2006) The development and evolution of division of labor and foraging specialization in a social insect (Apis mellifera L.). Curr Top Dev Biol 74:253-286. doi:10.1016/S0070-2153(06)74008-X

Pankiw T, Page RE Jr (1999) The effect of genotype, age, sex, and caste on response thresholds to sucrose and foraging behavior of honey bees (Apis mellifera L.). J Comp Physiol A 185(2):207-213

Pankiw T, Page RE (2000) Response thresholds to sucrose predict foraging division of labor in honeybees. Behav Ecol Sociobiol 47(4):265-267

Pankiw T, Page RE (2001) Genotype and colony environment affect honeybee (Apis mellifera L.) development and foraging behavior. Behav Ecol Sociobiol 51(1):87-94

Robinson GE (1985) Effects of a juvenile-hormone analog on honey bee foraging behavior and alarm pheromone production. J Insect Physiol 31(4):277-282

Robinson GE (1987) Regulation of honey-bee age polyethism by juvenile-hormone. Behav Ecol Sociobiol 20(5):329-338

Rutz W, Luscher M (1974) The occurrence of vitellogenin in workers and queens of Apis mellifica and the possibility of its transmission to the queen. J Insect Physiol 20(5):897-909

Seeley TD (1982) Adaptive significance of the age polyethism schedule in honeybee colonies. Behav Ecol Sociobiol 11(4):287-293

Seeley TD (1995) The wisdom of the hive: the social physiology of honey bee colonies. Harvard University Press, Cambridge

Seeley TD, Kolmes SA (1991) Age poleythism for hive duties in honeybees-illusion or reality? Ethology 87(3-4):284-297

Sekiguchi K, Sakagami SF (1966) Structure of foraging population and related problems in the honeybee, with consideration on the division of labour in honey bees. Hokkaido Agr Exp Sta Rep 69:1-65

Tofts C, Franks NR (1992) Doing the right thing — ants, honeybees and naked mole-rats. Trends Ecol Evol 7(10):346-349

Winston ML (1987) The biology of the honey bee. Harvard University Press, Cambridge

Winston ML, Katz SJ (1982) Foraging differences between crossfostered honeybee workers (Apis-mellifera) of European and Africanized races. Behav Ecol Sociobiol 10(2):125-129

Winston ML, Punnett EN (1982) Factors determining temporal division of labor in honeybees. Can J Zool 60(11):2947-2952 